André R. Miserez $\cdot$ Florence Clavaguera

Andreas U. Monsch · Alphonse Probst · Markus Tolnay

\title{
Argyrophilic grain disease: molecular genetic difference to other four-repeat tauopathies
}

Received: 3 April 2003 / Revised: 16 June 2003 / Accepted: 16 June 2003 / Published online: 29 August 2003

(C) Springer-Verlag 2003

\begin{abstract}
Argyrophilic grain disease (AgD) is a four-repeat tauopathy that is almost exclusively restricted to allocortical areas. Progressive supranuclear palsy and corticobasal degeneration also show predominant deposition of four-repeat tau filaments, and are associated with the tau H1 haplotype. We investigated a possible association between $\mathrm{AgD}$ and the tau $\mathrm{H} 1$ haplotype. In $\mathrm{AgD}$, no difference between the prevalence of the tau $\mathrm{H} 1$ haplotype or H1/H1 genotype was observed when compared to non-demented control cases. These data suggest that a dysfunction of the tau protein in $\mathrm{AgD}$ - in contrast to other fourrepeat tauopathies - may arise irrespective of the genetic background regarding the tau $\mathrm{H} 1$ or $\mathrm{H} 2$ haplotypes.
\end{abstract}

Keywords Argyrophilic grain disease $\cdot$ Four-repeat tauopathies · Tau H1 haplotype

\section{Introduction}

Argyrophilic grain disease $(\mathrm{AgD})$, first described by Braak and Braak $[2,3]$ is a sporadic late onset dementia that accounts for approximately $5 \%$ of all cases of dementia [5, $13,17]$. Among the oldest-old a recent study suggests that $\mathrm{AgD}$ is the second most common cause of degenerative dementia in Japan, after Alzheimer's disease (AD) [12]. Morphologically, $\mathrm{AgD}$ is characterized by the presence of

\section{A. R. Miserez}

Cardiovascular Genetics, Institute of Biochemistry and Genetics, Department of Clinical-Biological Sciences, University of Basel, Basel, Switzerland

F. Clavaguera $\cdot$ A. Probst $\cdot$ M. Tolnay (凶)

Department of Neuropathology, Institute of Pathology,

University of Basel, Schönbeinstrasse 40, 4003 Basel, Switzerland

Tel.: +41-61-2652525, Fax: +41-61-2653194,

e-mail: mtolnay@uhbs.ch

\section{A. U. Monsch}

Memory Clinics, Geriatric University, University of Basel, Basel, Switzerland neuronal argyrophilic grains (ArGs) in various limbic structures, including the hippocampus, the entorhinal cortex, and the amygdala, and by coiled bodies in oligodendrocytes $[2,3]$. Both filamentous lesions consist of the microtubule-associated protein tau in an abnormally hyperphosphorylated state [16]. Recent biochemical studies further revealed that tau filamentous inclusions in $\mathrm{AgD}$ consist primarily of tau isoforms with four microtubule-binding repeats (4R-tau) $[14,12,19]$.

The predominant deposition of 4R-tau is also a feature of progressive supranuclear palsy (PSP) and corticobasal degeneration (CBD) [7], and both tauopathies have been shown to be associated with the tau H1 haplotype [1, 6]. A recent study also suggests a trend towards an increased tau H1 haplotype in AgD. However, no statistically significant difference was found between $\mathrm{AgD}$ and non-tauopathy controls [14]. Based on these findings, we investigated the tau $\mathrm{H} 1$ haplotype in $\mathrm{AgD}$, and wanted to know whether the $4 \mathrm{R}$ tauopathies share a common pathogenic mechanism causing dysfunction of the tau protein. Tau haplotypes $\mathrm{H} 1$ and $\mathrm{H} 2$ were analyzed in a sample of 79 subjects with neuropathologically confirmed AgD according to published criteria $[5,13,17]$, and in a sample of 148 nondemented control subjects without ArGs.

\section{Material and methods}

AgD cases $(n=79)$ diagnosed at the Department of Neuropathology, Basel University, Switzerland were included in the present study. There were 33 males, 46 females, with a median age of 85.9 years (66-96 years). Standard neuropathological examination was performed including the Gallyas silver technique, and immunohistochemistry using antibodies against tau (AT8; 1:1,000; Innogenetics, Gent, Belgium), $\beta$-amyloid (1:50; Dako, Glostrup, Denmark), $\alpha$-synuclein $(1: 2,000$; Zymed, San Francisco, CA) and $\alpha \mathrm{B}$-crystallin (1:1,000; Novocastra, Newcastle, UK). As controls, 148 nondemented subjects were randomly selected from the Basel InterDisciplinary study on Aging (IDA), Switzerland [10]. For molecular analysis, paraffin-embedded tissue samples and/or blood samples from the $\mathrm{AgD}$ cases and blood samples from the controls were used.

Genomic DNA was extracted from tissue samples using DNeasy tissue kits from Qiagen (Hilden, Germany), and from nucleated 

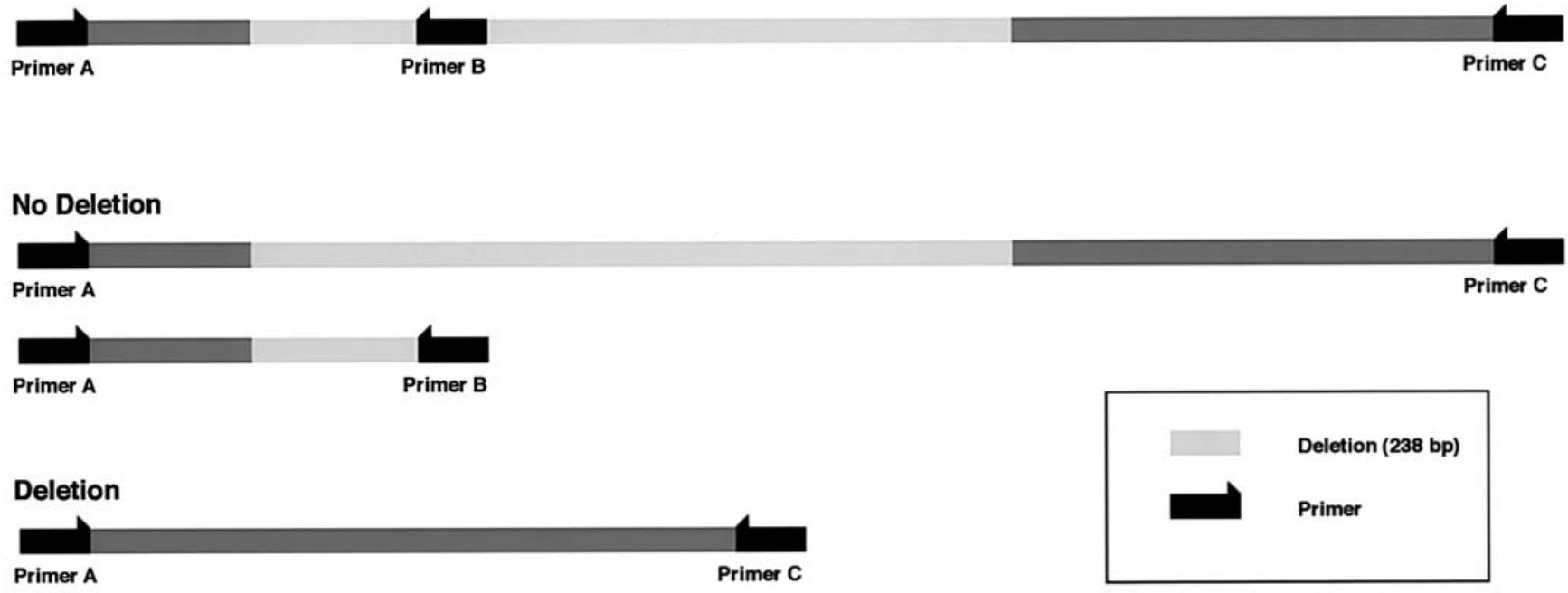

B

5 ' -ggaagacgttctcactgatctg-3' Primer A (sense, both alleles)

5' - -atgggtctcccacgactgcatt-3' Primer B (antisense, allele-specific)

5 '-aggagtctggcttcagtctctc-3. Primer C (antisense, both alleles) ggaagacgttctcactgatctggcagagaaaatgtccagtttttccaactccetaaaccatggttttctatttcatagttcttaggcaaattggtaaaaatcatttctcatcaaaacgetgatat

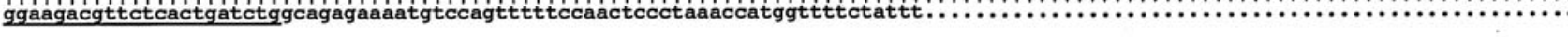
tttcacacctccetggtgtctgcagaaagaaccttccagaaatgcagtcgtgggagacccatccaggccaccctgcttatggaagagctgagaaaaagccccacgggagcatttgctcagcttc

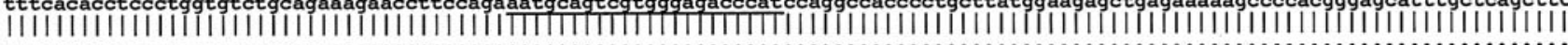
cqttacgcacctagtggcattgtgggtgggagagggctggtgggtggatggaaggagaaggcacagcceccettgcagggacagagccetcgtacagaagggacaccceacatttgtcttccec

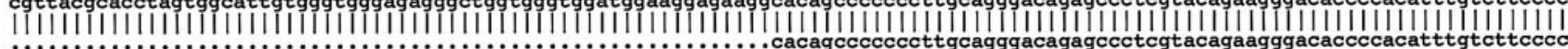

acaaagcggectgtgtcctgcctacggggtcagggcttctcaaacctggctgtgtgtcagaatcaccaggggaacttttcaaaactagagagactgaagccagactcct

|||||||||||||||||||||||||||||||||||||||||||||||||||||||||||||||||||||||||||||||||||||||||||||

Fig. 1 A Length of the DNA sequence amplified by the primers $\mathrm{A}, \mathrm{B}$, and $\mathrm{C}$ in the case of the presence or absence of the intronic deletion of $238 \mathrm{bp}$. B Sequence alignment of the two DNA sequences (presence and absence of the intronic 238 bp sequence)

blood cells by the salting out method with modifications as described [8], or using the QIAmp DNA blood kit. Taq DNA polymerase and deoxyribonucleotides were from Applied Biosystems (Norwalk, CT). Oligonucleotides were synthesized by Microsynth Inc. (Balgach, Switzerland); Spreadex EL 300 Wide-Mini S-100 gels were from Elchrom Inc. (Cham, Switzerland). To characterize the 238-bp deletion in intron 9, which corresponds to the $\mathrm{H} 2$ haplotype of the tau gene, the part of intron 9 containing the 238-bp deletion was amplified using primers as previously described [1]. The amplicon was separated on a $2 \%$ agarose gel, dissolved, purified, and subjected to dideoxy sequencing. The presence of the intronic deletion was determined by allele-specific PCR. To investigate the presence of the deletion in each sample, the allele-specific antisense primer tau-del9.asR, 5'-ATGGGTCTCCCACGACTGCATT-3' (primer B), complimentary to parts of the intron sequence of the deletion, was designed. Using all three primers [tau-del9F (A), taudel19.asR (B), and tau-del19.R (C)] in the same reaction combined (multiplex PCR) or pairs of primers $(\mathrm{A} / \mathrm{B}$ and $\mathrm{A} / \mathrm{C}$ ), the presence of the deletion could be determined for both alleles of the individual investigated. Using the primer combination $\mathrm{A} / \mathrm{C}$, a fragment of $484 \mathrm{bp}$ was obtained if the deletion was absent and a fragment of $246 \mathrm{bp}$ if the intronic sequence was deleted. However, as the probability of amplifying a fragment of $484 \mathrm{bp}$ is theoretically lower than the probability of amplifying a fragment of $246 \mathrm{bp}$, we also used the primer combination A/B. This PCR results in a fragment of $187 \mathrm{bp}$ if the intronic fragment is not deleted (Fig. 1A). For group comparison, the Chi-square statistic was used.

\section{Results}

Histologically, all AgD cases were characterized by the presence of abundant ArGs in limbic areas, including sector CA1 of the hippocampus, the entorhinal cortex and the amygdala. Coiled bodies were mainly found in the white matter underneath cortical regions rich in ArGs. AT8 revealed various numbers of pretangle neurons in all AgD cases as previously described [16]. In all cases, $\alpha \mathrm{B}$-crystallin-stained ballooned neurons were predominantly found in the amygdala, corroborating earlier results [15]. Associated neurofibrillary tangles of the AD type were found in a density and distribution corresponding to early transentorhinal or limbic Braak stages [Braak stage I, $17(21.5 \%)$ cases; Braak stage II, 45 (57.0\%) cases; Braak stage III, $17(21.5 \%)$ cases] [4]. Some diffuse $A \beta$ deposits were present in $40(50.6 \%)$ cases, while the remaining cases were totally devoid of senile plaques. In three cases there were additional Lewy bodies and Lewy neurites. Vascular le- 
Table 1 Tau haplotype and genotype frequency in neuropathologically confirmed cases of argyrophilic grain disease and healthy control subjects

\begin{tabular}{lrrrr}
\hline & \multicolumn{2}{c}{$\begin{array}{l}\text { Argyrophilic grain disease } \\
n=79 \text { (cases) }\end{array}$} & \multicolumn{2}{c}{$\begin{array}{l}\text { Control subjects } \\
n=148 \text { (cases) }\end{array}$} \\
\hline Alleles & & & & \\
H1 & 122 & $(77.2 \%)$ & 228 & $(77.0 \%)$ \\
H2 & 36 & $(22.8 \%)$ & 68 & $(23.0 \%)$ \\
Genotypes & & & & \\
H1/H1 & 46 & $(58.2 \%)$ & 89 & $(60.1 \%)$ \\
H1/H2 & 30 & $(38.0 \%)$ & 50 & $(33.8 \%)$ \\
H2/H2 & 3 & $(3.8 \%)$ & 89 & $(6.1 \%)$ \\
\hline
\end{tabular}

sions (e.g., small lacunar infarcts and/or cribriform state of the basal ganglia) were found in $19(24.1 \%)$ cases. All $\mathrm{AgD}$ cases were devoid of concomitant PSP and CBD pathology. The neuropathological findings of 24 cases have been reported in earlier studies [17]. According to the clinical records, $61(77.2 \%)$ subjects with ArGs were reported to be demented.

Figure 1B presents the sequence containing the 238-bp deletion as well as the localization of the deletion itself. Molecular analysis revealed the following tau genotype frequencies for the $\mathrm{AgD}$ cases: genotype $\mathrm{H} 1 / \mathrm{H} 146 \mathrm{pa}-$ tients $(58.2 \%)$, genotype $\mathrm{H} 1 / \mathrm{H} 230$ patients $(38.0 \%)$, and genotype $\mathrm{H} 2 / \mathrm{H} 23$ patients $(3.8 \%)$. The tau genotype frequencies of the non-demented control group did not differ significantly from that of the $\mathrm{AgD}$ cases $(P=0.672)$. Hence, among the $\mathrm{AgD}$ cases, the $\mathrm{H} 1$ allele was found in $77.2 \%$ and the $\mathrm{H} 2$ allele in $22.8 \%$, respectively. These values did not differ significantly from those obtained in the non-demented control group $(P=0.964)$ (Table 1$)$.

\section{Discussion}

PSP and CBD are sporadic extrapyramidal syndromes characterized by predominant 4R tau deposition, and both have been shown to be associated with the tau H1 haplotype $[1,6]$. The sporadic late-onset dementia AgD has recently been recognized as a $4 \mathrm{R}$ tauopathy $[14,18,19]$. Preliminary data based on a limited number of cases opened the possibility that the tau H1 haplotype might also be overrepresented in AgD [14]. To test the hypothesis whether the association of the tau H1 haplotype represents a common feature among $4 \mathrm{R}$ tauopathies, we analyzed the tau haplotype in a cohort of 79 autopsy-confirmed $\mathrm{AgD}$ cases. In contrast to PSP and CBD [1, 6], no difference was found between the prevalence of the tau $\mathrm{H} 1$ haplotype or the $\mathrm{H} 1 / \mathrm{H} 1$ genotype in subjects with $\mathrm{AgD}$ when compared to non-demented control cases.

The tau haplotype frequencies may vary when they are studied in different populations. The tau $\mathrm{H} 1 / \mathrm{H} 1$ genotype is known to be very low in Norway (50\%), while it is very high in Japan (>95\%). In our study, both the AgD and the control cohorts are derived from the same urban-suburban area in a northwestern region of Switzerland. In the control cohort the frequencies of the tau H1 haplotype (77.0\%) and $\mathrm{H} 1 / \mathrm{H} 1$ genotype $(60.1 \%)$ are quite comparable with values reported in different US- and UK-based populations $[1,6,9,14]$.

The present study suggests that there is no obligatory association between the tau H1 haplotype and the 4R tauopathies. Thus, the presence of the tau H1 haplotype seems not to be a prerequisite for the type of filamentous tau protein deposited in 4R tauopathies. No association between the tau H1 or H2 haplotype has been found in Pick's disease $[9,11]$, a tauopathy characterized by the predominant deposition of 3R tau filaments.

PSP, CBD and AgD share pathological and biochemical similarities with regard to an abnormal tau deposition. In all these disorders, predominantly $4 \mathrm{R}$ tau filamentous inclusions are found in neurons, astrocytes and oligodendrocytes. A recent study further demonstrates a high prevalence of ArGs in PSP and CBD cases [14]. In contrast, a high prevalence of PSP and CBD cases has not been observed in $\mathrm{AgD}$ cases, even in large cohorts of cases [5, 17]. Other differences, however, may confirm the separation of PSP and CBD from $\mathrm{AgD}$ as distinct clinicopathological entities. Clinically, PSP patients present with postural instability, supranuclear vertical gaze palsy, parkinsonism, pseudobulbar palsy and subcortical dementia. CBD patients also exhibit motor symptoms, parkinsonism and eye movement abnormalities, and dementia has been reported as their presenting syndrome in the majority of CBD patients. In contrast, although the clinical characteristics of $\mathrm{AgD}$ remain to be fully established, motor symptoms and parkinsonism are not characteristic features of $\mathrm{AgD}$. Recent studies rather suggest that $\mathrm{AgD}$ patients present with dementia, and that personality changes and emotional imbalances may precede memory failure [17]. The different clinical presentation among 4R tauopathies is a function of the anatomical distribution of their pathology. Thus, PSP and CBD are characterized by a widespread tau pathology in various cortical and subcortical structures, including brain stem, while tau filamentous lesions in $\mathrm{AgD}$ are almost exclusively restricted to allocortical areas. Whether the tau haplotypes may affect the different topology of neurodegeneration among 4R tauopathies remains to be elucidated.

In conclusion, the present study further establishes $\mathrm{AgD}$ as a rather distinct pathological entity within the heterogeneous group of tauopathies. Hence, the clinicopathological differences between the 4R tauopathies PSP and CBD on one hand and $\mathrm{AgD}$ on the other might be explained by genetic or non-genetic differences that are involved in the development of the tau protein dysfunction.

Acknowledgements This work was supported by the Basel Foundation for Dementia Research (to A.R.M. and M.T.) and by the Swiss National Science Foundation (SNSF) (A.R.M.; grant number 3200-049125.96/1). A.R.M. is supported by the Swiss Clinicians Opting for Research (SCORE), a grant number 3231048896.96 of the SNSF. F.C is supported by the SNSF (grant number 3100-068328). We thank Michelle Pfeifer for help with the manuscript. 


\section{References}

1. Baker M, Litvan I, Houlden H, Adamson J, Dickson D, PerezTur J, Hardy J, Lynch T, Bigio E, Hutton M (1999) Association of an extended haplotype in the tau gene with progressive supranuclear palsy. Hum Mol Genet 8:711-715

2. Braak H, Braak E (1987) Argyrophilic grains: characteristic pathology of cerebral cortex in cases of adult onset dementia. Neurosci Lett 76:124-127

3. Braak H, Braak E (1989) Cortical and subcortical argyrophilic grains characterize a disease associated with adult onset dementia. Neuropathol Appl Neurobiol 15:13-26

4. Braak H, Braak E (1991) Neuropathological stageing of Alzheimer-related changes. Acta Neuropathol 82:239-259

5. Braak H, Braak E (1998) Argyrophilic grain disease: frequency of occurrence in different age categories and neuropathological diagnostic criteria. J Neural Transm 105:801-819

6. Houlden H, Baker M, Morris HR, et al (2001) Corticobasal degeneration and progressive supranuclear palsy share a common tau haplotype. Neurology 56:1702-1706

7. Lee VM-Y, Goedert M, Trojanowski JQ (2001) Neurodegenerative tauopathies. Annu Rev Neurosci 24:1121-1159

8. Miserez AR, Laager R, Chiodetti N, Keller U (1994) High prevalence of familial defective apolipoprotein B-100 in Switzerland. J Lipid Res 35:574-583

9. Morris HR, Baker M, Yasojima K, et al (2002) Analysis of tau haplotypes in Pick's disease. Neurology 59:443-445

10. Perrig-Chiello P, Perrig WJ, Stähelin HB, Krebs-Roubicek E, Ehrsam R (1996) Wellbeing, health and autonomy in old age: the Basel IDA Study (Interdisciplinary Aging Study). Z Gerontol Geriatr 29:95-109

11. Russ C, Lovestone S, Baker M, Pickering-Brown SM, Andersen PM, Furlong R, Mann D, Powell JF (2001) The extended haplotype of the microtubule associated protein tau gene is not associated with Pick's disease. Neurosci Lett 299:156-158
12. Saito Y, Nakahara K, Yamanouchi H, Murayama S (2002) Severe involvement of ambient gyrus in dementia with grains. J Neuropathol Exp Neurol 61:789-796

13. Togo T, Cookson N, Dickson DW (2002) Argyrophilic grain disease: neuropathology, frequency in a dementia brain bank and lack of relationship with apolipoprotein E. Brain Pathol $12: 45-52$

14. Togo T, Sahara N, Shu-Hui Y, Cookson N, Ishizawa T, Hutton M, De Silva R, Lees A, Dickson DW (2002) Argyrophilic grain disease is a sporadic 4-repeat tauopathy. J Neuropathol Exp Neurol 61:547-556

15. Tolnay M, Probst A (1999) Ballooned neurons expressing alphaB-crystallin as a constant feature of the amygdala in argyrophilic grain disease. Neurosci Lett 246:165-168

16. Tolnay M, Spillantini MG, Goedert M, Ulrich J, Langui D, Probst A (1997) Argyrophilic grain disease: widespread hyperphosphorylation of tau protein in limbic neurons. Acta Neuropathol 93:477-484

17. Tolnay M, Monsch AU, Probst A (2001) Argyrophilic grain disease: a frequent dementing disorder in aged patients. Adv Exp Med Biol 487:39-58

18. Tolnay M, Sergeant N, Ghestem A, Chalbot S, Vos RAI de, Jansen Steur ENH, Probst A, Delacourte A (2002) Argyrophilic grain disease and Alzheimer's disease are distinguished by their different distribution of tau protein isoforms. Acta Neuropathol 104:425-434

19. Zhukareva V, Shah K, Uryu K, Braak H, Del Tredici K, Sundarraj S, Clark C, Trojanowski JQ, Lee VM (2002) Biochemical analysis of tau proteins in argyrophilic grain disease, Alzheimer's disease, and Pick's disease. A comparative study. Am J Pathol 161:1135-1141 